\section{EZH2 MODULATES THE DNA METHYLOME AND CONTROLS LYMPHOCYTE ADHESION THROUGH JUNCTIONAL ADHESION MOLECULE-A: IMPLICATION OF ITS PATHOGENIC ROLE IN SLE}

PS Tsou, P Coit, S Miller, A Sawalha*. University of Michigan, Internal Medicine, Ann Arbour, USA

10.1136/lupus-2017-000215.312

Background and aims EZH2 is an epigenetic regulator that trimethylates lysine 27 of histone 3 (H3K27me3). We have previously suggested that increased EZH2 might be mediating a pro-inflammatory epigenetic reprograming of naïve $\mathrm{CD} 4+\mathrm{T}$ cells as an early event in lupus flares. Here we examined how overexpression of EZH2 affects the DNA methylome and function in $\mathrm{CD} 4+\mathrm{T}$ cells.

Methods Naïve CD4+ $\mathrm{T}$ cells were isolated from lupus patients and healthy controls. EZH2 was overexpressed, and genome-wide DNA methylation changes were evaluated. Gene expression and miRNAs were assessed by qPCR while protein expression was examined by Western blotting. A cell adhesion assay was used to assess adhesion of $\mathrm{T}$ cells to human microvascular endothelial cells (HMVEC).

Results EZH2 expression and $\mathrm{H} 3 \mathrm{k} 27 \mathrm{me} 3$ were increased in naïve CD4+ $\mathrm{T}$ cells in lupus compared to healthy controls. Both miR-26a and miR-101, which regulate EZH2, were decreased. DNA methylation analysis identified 156 hypomethylated and 168 hypermethylated $\mathrm{CpG}$ sites in naïve CD4+ $\mathrm{T}$ cells transfected with EZH2. Genes involved in leukocyte adhesion and migration, such as F11R encoding JAM-A (junctional adhesion molecule A), and SELPLG encoding PSGL-1 (P-selectin glycoprotein ligand 1), were hypomethylated. Overexpression of EZH2 resulted in $\sim 2$-fold increased adhesion of CD4+ $\mathrm{T}$ cells to endothelial cells. Pre-incubation of EZH2transfected CD4 $+\mathrm{T}$ cells with neutralising antibodies against JAM-A significantly blunted cell adhesion.

Conclusions We uncovered an important role for EZH2 in $\mathrm{T}$ cell adhesion. EZH2 overexpression results in hypomethylation of JAM-A, which might increase the migratory ability of $\mathrm{T}$ cells and contribute to aggressive $\mathrm{T}$ cell extravasation in lupus.

\section{VERY RARE X CHROMOSOME ANEUPLOIDIES IN LUPUS AND SJOGREN'S}

${ }^{1} \mathrm{H}$ Scofield ${ }^{*},{ }^{2} \mathrm{R}$ Sharma, ${ }^{3} \mathrm{~V}$ Harris, ${ }^{4} \mathrm{~J}$ Cavett, ${ }^{5} \mathrm{~J}$ Harley, ${ }^{4} \mathrm{~B}$ Kurien, ${ }^{6} \mathrm{~A}$ Rasmussen, ${ }^{6} \mathrm{~K}$ Sivils for SGENE. ' USA; ' University of Oklahoma Health Science Centre, Medicine, Oklahoma City, USA; ${ }^{3}$ University of Oklahoma Health Sciences Centre, Pathology, Oklahoma City, USA; ${ }^{4}$ University of Oklahoma Health Sciences Centre, Medicine, Oklahoma City, USA; ${ }^{5}$ Cincinnati Children's Hospital Medical Center-University of Cincinnati, Centre for Autoimmune Genomics and Etiology-, Cincinnati, USA; ${ }^{6}$ Oklahoma Medical Research Foundation, Arthritis and Clinical Immunology, Oklahoma City, USA

\subsection{6/lupus-2017-000215.313}

Background and aims Systemic lupus erythematosus (SLE) and Sjögren's syndrome are related by clinical and serological manifestations as well as genetic risks. Both diseases are more commonly found in women compared to men at a ratio of about 10 to 1 . Common X chromosome aneuploidies, 47, XXY and 47,XXX, are enriched among men and women, respectively, in either disease suggesting a dose effect on the $\mathrm{X}$ chromosome.
Methods We examined cohorts of Sjögren's syndrome or SLE patients with intensity plots of $\mathrm{X}$ chromosome single nucleotide polymorphism (SNP) alleles along with karyotype of selected subjects.

Results Among $\sim 2500$ women with SLE we found three patients with a triple mosaic consisting of $45, \mathrm{X} / 46, \mathrm{XX} / 47$, XXX. Among $\sim 2100$ women with Sjögren's syndrome, one patient had $45, \mathrm{X} / 46, \mathrm{XX} / 47, \mathrm{XXX}$ with a triplication of the distal $\mathrm{p}$ arm of the $\mathrm{X}$ chromosome in the 47,XXX cells. Neither the triple mosaic nor the partial triplication were found among controls. In another Sjögren's cohort, we found a mother-daughter pair with partial triplication of this same region of the $\mathrm{X}$ chromosome. The triple mosaic occurs in approximately 1 in 25000 to 50000 live female births, while partial triplications such are even rarer.

Conclusions Very rare $\mathrm{X}$ chromosome abnormalities are present among patients with either Sjögren's or SLE, and may inform the location of a gene(s) that mediate an X dose effect as well as critical cell types in which such effect is operative.

\section{ROLE OF CYTOKINE IL-5 AND IL-25 AS BIOMARKERS IN SYSTEMIC LUPUS ERYTHEMATOSUS}

${ }^{1} \mathrm{M}$ Selvaraja*, ${ }^{1} \mathrm{M}$ Abdullah, ${ }^{2} \mathrm{~A}$ Md Shah, ${ }^{3} \mathrm{MB}$ Arip, ${ }^{4} \mathrm{~S}$ Amin Nordin. ${ }^{1}$ Universiti Putra Malaysia, Department of Pathology, Serdang, Malaysia; '2Universiti Putra Malaysia, Department of Medicine, Serdang, Malaysia; ${ }^{3}$ Institute for Medical Research, Autoimmune Unit, Kuala Lumpur, Malaysia; ${ }^{4}$ Universiti Putra Malaysia, Department of Medical Microbiology and Parasitology, Serdang, Malaysia

\subsection{6/lupus-2017-000215.314}

Background and aims Systemic Lupus Erythematosus (SLE) is a chronic and prototypic multisystem autoimmune disease which present with extensive clinical features affecting almost every organs and tissues. At current situation, the exact cause of aetiology remains unknown, however besides genetic, environmental and other hormonal factors, faulty in the immunological system include T- and B-Cell abnormalities and the failure to clear autoantibodies which causes generation of immune complex and disparity in the level of cytokine also have been described in SLE leading to trigger inflammation and induce organ damage. There are various key cytokines been studied and identified to be therapeutic target for SLE.

Methods In the present study, we investigated on the level of IL-25, IL-35, IL-2, IL-4, IL-5, IL-6, IL-8 and IL-10 among Malaysian Malay female SLE population and the possible association to disease severity leading to lupus nephritis. In the present study, SLE group divided into two categories, one is SLE with Lupus Nephritis (SLE-LN) and one with SLE alone comparing to normal control. ELISA method were used to measure the level of cytokines.

Results From the results, we found that SLE-LN and SLE had the higher level IL-35, IL-25, IL-8 and IL-10 but lower level of IL-5, IL-2 and IL-6 compared to normal control with IL-4 have no detection. IL-5 and IL-2 significantly $(p<0.005)$ inhibited among SLE and SLE-LN while IL-25 significantly elevated in SLE and SLE-LN compared to control.

Conclusions This suggest IL-5 and IL-25 as beneficial marker for SLE disease activity. 